\title{
RECHERCHES SUR LE CYCLE ÉVOLUTIF DES CYLINDROTAENIA
}

\author{
Par Ch. JOYEUX
}

Le genre Cylindrotænia a été créé par Minna E. Jewel (1916) pour un cestode, $C$. americana n. sp., récolté chez divers batraciens anoures aux Etats-Unis. On trouvera dans le travail de cet auteur tous les caractères différenciant son nouveau genre de Nematotænia proposé par Lühe (1899) pour Tænia dispar Göze, 1782, dont une bonne description a été donnée par O. Fuhrmann (1895). Ce dernier est très commun chez les batraciens de divers pays, notamment du bassin méditerranéen. M. E. Jewel a enlevé Nematotænia aux Paruterinæ Fuhrmann pour le placer, avec Cylindrotænia, dans une nouvelle sous-famille : Cylindrotænianæ (1), subdivision de la famille des Dilepinidæ Fuhrmann. Tænia pulchella Leidy, 1851, de Bufo viridis, appartient probablement à cette sous-famille, dont voici la diagnose d'après M. E. Jewel (1916) : "Cylindrical Dilepinidx having one or two dorsally placed testes, ovary and vitellaria dorsal to ovary. Proglottids distinct at the posterior end only. The uterus breaks down early and the embryos are later enclosed in para-uterine capsules. "

Ultérieurement Lloyd B. Dickey (1921) a créé le genre Distoichometra, avec D. bufonis n. sp. comme type, pour un ténia de Bufo lentiginosus, récolté aux Etats-Unis, qu'il range à côté des deux précédents. La sous-famille des Cylindrotæniinæ comprend donc actuellement les trois genres Cylindrotænia, Nematotænia et Distoichometra. Je renvoie aux travaux de M. E. Jewel et L. B. Dickey pour l'étude de leurs caractères différentiels, me bornant à donner ici la diagnose de Cylindrotænia qui nous intéresse spécialement : " Scolex unarmed, without rostellum ; reproductive organs single in each proglottid; pores lateral, alternating ; vagina and cirrus dorsal to the excretory canals and main nerve trunk; testis one, dorsal ; ovary and vitellaria ventral. Uterus breaks into capsules surrounding the embryos wich ultimately pass into two para-uterine capsules. " A ma connaissance, il n'existe qu'une seule espèce de

(1) Le terme correct est Cylindrotaniina.

Annales de Parasitologie, T. II, N $\mathrm{N}^{\circ}$ 1. - Janvier 1924, p. 74. 
Cylindrotænia, celle décrite comme type du genre par M. E. Jewel : C. americana.

J'ai eu l'occasion de retrouver récemment ce ténia chez des grenouilles dont je donnerai la détermination plus loin, capturées en Afrique du sud, aux environs de Lourenço-Marques (Mozambique) (1).

Le ver en question rentre incontestablement dans le genre Cylindrotænia : le seul point embarrassant est de savoir s'il faut l'identifier à $C$. americana ou en faire une espèce nouvelle. Le tableau suivant indique les caractères donnés par M. E. Jewel et, en regard, ceux que j'ai moi-même observés. A moins d'indications contraires, les chiffres représentent des $\mu$.

M. E. Jewel a trouvé des différences de taille chez les vers provenant de divers hôtes : Acris gryllis et Rana pipiens ; dans les cas d'infestation intense les parasites sont plus petits.

On remarquera, en outre, dans le tableau qui suit, que les divergences entre nos deux descriptions se rapportent surtout aux dimensions des parties molles. Dans mes échantillons, la longueur est moindre, le scolex et les ventouses plus larges, le cou plus court, les anneaux plus larges, l'utérus et l'organe para-utérin plus rapprochés de la tête. Toutes ces différences peuvent être mises sur le compte de la contraction.

Ce ver paraît d'ailleurs subir d'énormes variations de taille suivant son état de contraction ou d'extension, si l'on en juge par les formęes de développement que nous étudierons plus loin.

Par contre, les caractères fixes des échantillons de M. E. Jewel et des miens présentent peu de différences importantes : diamètre des vaisseaux aquifères, longueur de la poche du cirre, dimensions des cellules ovariennes, nombre d'œufs dans chaque anneau mûr, taille de ces œufs. La structure générale est absolument superpo. sable; on le constate soit en examinant par transparence les anneaux, examen difficile par suite de leur contraction; soit, plus facilement, en pratiquant des coupes sériées transversales.

M'appuyant, d'une part sur ces données, d'autre part sur les remarques de Jewel relatives aux différences de taille dans ces

(1) Cette récolte a été faite pendant une excursion organisée par le Gouvernement et le Service de Santé de Mozambique, en l'honneur des personnes ayant assisté au Congrès de Médecine tropicale de l'Angola. Au cours de divers articles parus dans la Presse médicale (novembre et décembre 1923) nous avons eu l'occasion, le professeur E. Brumpt et moi, de dire toute la cordialité de la réception qui nous a été faite par nos collègues portugais. Je leur renouvelle ici encore tous nos remerciements, spécialement au Dr Herminio Gomes, directeur de l'hôpital de Lourenço-Marques, grâce auquel j'ai pu me procurer le matériel étudié dans ce travail. 
DIMEMSIONS COMPARÉES DES EXEMPLAIRES AMÉRICAINS ET AFRICAINS

Longueur des vers ..........

Couleur....................

Coupe transversale..........

Diamètre transversal du scolex

Diamètre des ventouses......

Dimensions du cou...........

Largeur des premiers anneaux au début de la formation des organes génitaux....... Largeur des anneaux sexués..

Début de l'utérus............

Formation de l'organe parautérin....................

Dimensions des anneaux à ce niveau ..................

Dimensions des derniers anneaux ..................

Dimensions des anneaux mûrs détachés........................

Diamètre des vaisseaux aquifères au niveau des anneaux sexués ...................

Dimensions du testicule......

Dimensions de la poche du cirre.....................

Dimensions de l'ovaire.......

Dimensions des cellules ovariennes ......................

Dimensions des vitellogènes. . Diamètre de l'organe para-utérin ..... Diamètre des portions apicales sphériques de cet organe... Nombre d'œufs dans chaque anneau mûr................

Diamètre des œufs ...........

\begin{tabular}{c} 
EXEMPLAIRES \\
DE M.-E. Jewel \\
\hline 25 à 40 millimètres \\
maximum 80 millimètres \\
uniformément blanche \\
cylindrique \\
160 à 200
\end{tabular}

$2 / 5$ à $1 / 2$ du scolex

$5000 / 130$ à 150

134

$162 / 9$

11 millimètres après le scolex.

22 millimètres après le scolex

40 à $45 / 180$ à 200 et 350 si contraction

146 à $178 / 72$ à 74

$340 / 250$

5 à 7

$34 / 26$

36 à $44 / 13$ à 17

24 à 34

9

18

$130 / 124$

20

8 à 12

20
EXEMPLAIRES

PERSONNELS

15 millimètres

uniformément blanche cylindrique 550

160 (à peu près $1 / 3$ du scolex) $860 / 275$

\section{0}

$330 / 20$

5 millimètres après le scolex

7 millimètres après le scolex

$350 / 80$

250 à $320 / 160$ à 235

$400 / 250$ (sur le point de se détacher)

8 à 10

$60 / 40$ aplati

$40 / 28$

$40 / 30$ aplati

10

22

$132 / 85$

45

10 à 12

25 
divers spécimens, je crois pouvoir me dispenser de créer une nouvelle espèce et, dans l'état actuel de nos connaissances, identifier mes cestodes à Cylindrotænia americana Jewel, 1916.

Evolution. - M. E. Jewel signale, dans l'intestin des batraciens autopsiés, à côté des ténias adultes, de petits vers n'ayant qu'ur millimètre et demi de long. J'ai observé la même chose ; de plus, en examinant à un faible grossissement l'intestin des grenouilles, j'ai découvert, dans l'épaisseur de la muqueuse, diverses formes larvaires correspondant incontestablement au cestode en question.

Voici comment on peut concevoir le cycle évolutif de ce ténia. Il doit s'accomplir directement, sans hôte intermédiaire, comme celui de l'Hymenolepis fraterna du rat. Je n'ai pu suivre le début du développement : il est probable que l'œuf, ou l'anneau mûr contenant les œufs, expulsé avec les matières fécales d'un batracien parasité, est avalé par un autre batracien. Dans l'intestin de ce dernier, les embryons hexacanthes sont mis en liberté ef vont se loger dans la muqueuse; là ils grandissent. Le premier stade observé (fig. 1) montre un embryon hypertrophié, ayant déjà perdu ses crochets hexacanthes. Cette perte prématurée rend difficile la recherche des jeunes parasites qui, ne possédant plus ces organes, précieux points de repère, m'ont peut-être échappé. L'embryon est à l'intérieur d'un kyste, sans doute formé par réaction de l'hôte, ainsi que cela a lieu pour certains cysticercoïdes, notamment ceux de Dipylidium évoluant chez des reptiles. Sa structure est extrêmement simple : point de cavité lacunaire comme chez les larves de ce type, mais simplement une masse de parenchyme non différenciée. On ne voit pas encore l'ébauche des organes de la tête.

A un stade suivant (fig. 2), le cystique est encore dans son kyste ; il a grandi.

Le pôle caudal s'est allongé et montre une queue épaisse qui est repliée dans le kyste ; l'autre pôle s'est différencié, il présente les ébauches des ventouses, apparaissant avant l'invagination, comme chez tous les cysticercoïdes.

Je n'ai pu trouver le stade suivant : on peut d'ailleurs facilement l'imaginer. Il y a sans doute invagination du scolex dans le reste du corps. Cette opération est gênée par la consistance des tissus, à cause de l'absence de cavité lacunaire ; la queue se détache également, en même temps la larve s'échappe du kyste qui la contenait.

Le cysticercoïde est alors mûr (fig. 3 et 4)! D'aspect piriforme, son extrémité caudale présente, comme les cysticercoïdes de Dipylidium, une échancrure qui disparaît ensuite, correspondant à l'insertion 
de l'appendice caudal disparu. Il n'existe aucune différenciation de la paroi, comme chez les cystiques d'Hymenolepis, pas d'involucre (paroi externe) destiné à dégénérer. Comme chez les Dipylidium, la larve tout entière contribuera à former l'adulte. Le rostre étant inerme et rudimentaire, il n'y a pas d'invagination secondaire destinée à le contenir. En un mot, le cysticercoïde est composé d'une simple masse de parenchyme dont le pôle antérieur, correspondant au futur scolex, s'est invaginé incomplètement dans le reste du corps. Je ne crois pas qu'on ait décrit, jusqu'à présent, de cysticercoïde ayant une constitution aussi rudimentaire.

Dans leur étude des cystiques, Grassi et Rovelli (1892) proposent la classification suivante :

$1^{\circ}$ Cystiques à invagination initiale, sans involucre embryonnaire: Archigetes. L'invagination n'est pas encore acquise et peut se faire à la volonté de l'animal.

$2^{\circ}$ Cystiques à invagination tardive, comprenant peut-être tous les cysticercoïdes ; ce groupe se divise en deux :

A. Invagination simple : Dipylidium caninum (L.).

B. Invagination suivie de la formation d'un involuere: Hymenolepis.

J'ai déjà décrit (1920, p. 196) un eysticercoïde de Geotrupes sylvaticus Panzer (Coléoptères), possédant une structure analogue à celle de la larve que je viens d'étudier, mais semblant n'avoir jamais possédé de queue. Sa structure est toutefois un peu plus compliquée que celle du cystique de Cylindrotænia, car, pendant son développement, existe une cavité lacunaire, ce qui permet à l'invagination de se faire complètement. Le scolex s'enfonce dans cette cavité, entièrement vide ou vaguement encombrée de lâches travées de mésenchyme, qu'il déprime; il est entièrement recouvert par le reste du corps de la larve. C'est donc une forme comparable aux cysticercoïdes de Dipylidium, moins la queue. Chez Cylindrotænia, au contraire, l'absence de cavité et la consistance parenchymateuse de la larve rendent l'invagination plus difficile, cette opération étant gênée par la résistance des tissus. Il s'ensuit qu'elle est moins complète que dans les autres types (1).

Ces deux larves: celle de Cylindrotænia d'abord, celle trouvée chez G. sylvaticus ensuite, doivent être placées, dans la classification

(1) Je renvoie à mon travail de 1920 pour tous les détails sur la structure de ces divers types de cysticercoïdes. J'y avais émis l'hypothèse que le cystique de G. sylvaticus était une forme nouvelle ou correspondait peut-être à un anoplocéphalidé : Monieza expansa (Rud.). A la suite de nombreuses expériences que je relaterai ultérieurement, je crois pouvoir affirmer que ce cystique n'a rien à faire avec l'évolution de Moniezia expansa. 
de Grassi et Rovelli, entre Archigetes et Dipylidium, c'est-à-dire à la base du rameau phylogénétique des cysticercoïdes.

Le reste de l'évolution de notre cestode est facile à suivre. Le scolex s'évagine et la partie postérieure du cystique s'allonge, donnant le cou du futur ténia (fig. 5) ; en même temps les ventouses
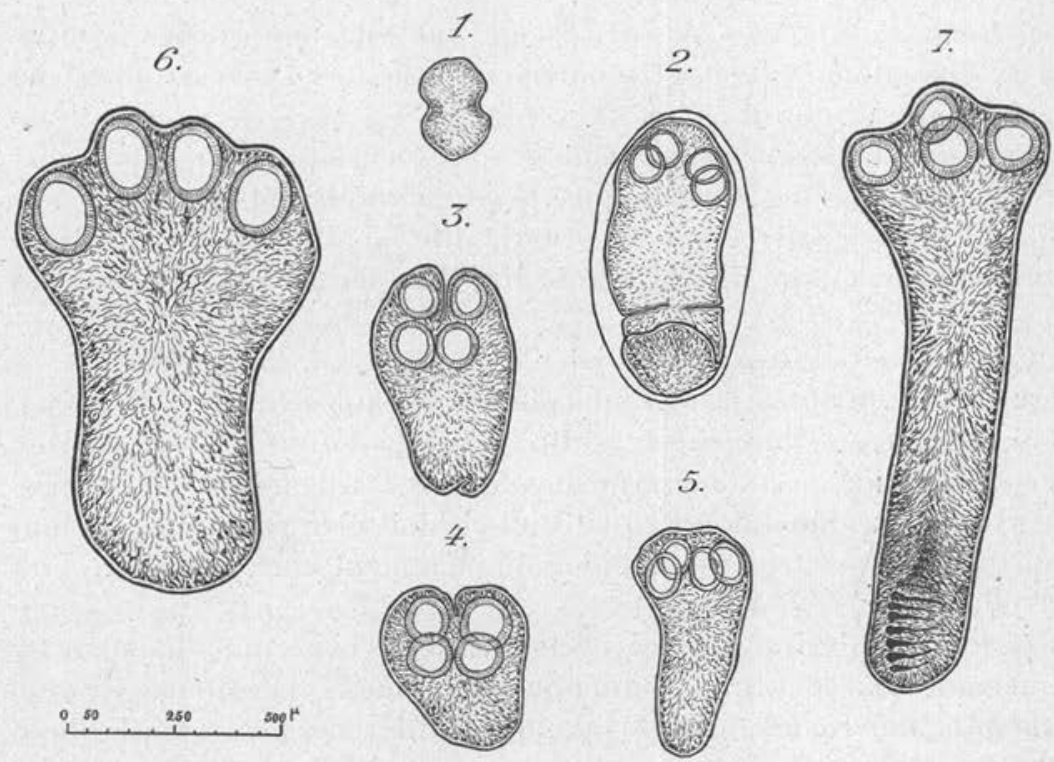

Fı́. - 1, Cysticercoïde en formation extrait de son kyste. Très contracté : le kyste ovalaire dont il a été énucléé mesure 1 millimètre sur $0 \mathrm{~mm}$., 5 . Ses dimensions à l'état frais sont donc à peu près triples de celles figurées ici ; 2, Cysticercoïde en formation dans son kyste. Ebauche des ventouses et développement du pôle caudal ; 3, Cysticercoïde mûr présentant, à la partie postérieure, une échancrure correspondant à l'insertion de l'appendice caudal disparu ; 4, Cysticercoïde mûr; 5 , Cysticercoïde évaginé, commençant à s'allonger ; 6, Jeune ver avec cou développé ; 7, Jeune ver montrant déjà des ébauches sexuelles. Toutes nos figures sont dessinées au même grossissement. Noter les diftérences de taille suivant l'état de contraction.

se fixent à la muqueuse et le ver grandit. Dans le matériel que j'ai examiné, on voyait un grand nombre de ténias de tailles différentes, à des degrés divers de développement (fig. 6 et 7 ), depuis les très jeunes formes jusqu'aux vers adultes à anneaux mûrs.

Epidémiologie. - La récolte du matériel a été faite, comme nous l'avons vu, dans un ruisseau des environs de Lourenço-Marques (Mozambique), le 29 août et le 2 septembre 1923 , c'est-à-dire à la fin de la saison sèche. 
Il a été examiné :

55 têtards non déterminables exactement, probablement Rani$d æ(1)$, tous indemnes.

14 Arthroleptis sp. indemnes.

1 Arthroleptis ogoensis Boulenger adulte, montrant un cystique en voie de développement.

6 Rana æquiplicata Werner, d'âge moyen, non encore adultes. Deux étaient parasitées et montraient à la fois des cystiques, des vers jeunes et adultes.

Cylindrotænia americana peut donc se développer au moins chez deux batraciens de Mozambique : Rana rquiplicata et Arthroleptis ogoensis. Ce dernier étant de petite taille, il est probable que si le ténia devient adulte dans son intestin, il ne doit avoir qu'une faible longueur.

Ce cestode paraît ne pas exister chez le têtard, l'infestation ne se ferait done qu'après la métamorphose du batracien.

Je n'ai aucune donnée sur la durée de l'évolution : le fait de trouver chez le même hôte des formes à tous les degrés de développement indique sans doute que celui-ci doit être rapide. Rappelons qu'Hymenolepis fraterna évolue complètement chez le rat en 14 à 20 jours.

Il serait à désirer que ce cycle fût suivi expérimentalement en Amérique. J'ai identifié, comme on l'a vu, mes échantillons à Cylindrotænia americana, malgré quelques différences de détail. Peutêtre l'étude du développement en montrerait d'autres; il y aurait lieu de sêparer alors les espèces américaine et africaine en distinguant par la biologie ce que la morphologie seule est impuissante ì faire. Cette expérience ne paraît pas, a priori, difficile à réaliser. Il suffirait de faire avaler des anneaux mûrs de $C$. americana par des grenouilles élevées au laboratoire, à l'abri de toute contamination.

\section{RÉSUMÉ}

Cylindrotænia americana Jewel, 1916, cestode parasite des batraciens, décrit aux Etats-Unis, est signalé au Mozambique.

Il évolue directement, sans hôte intermédiaire, dans la paroi intestinale du batracien, laquelle réagit par la formation d'une membrane adventice.

(1) La détermination des batraciens est due à M. F. Angel, préparateur au Museum national d'Histoire Naturelle, je lui adresse de vifs remerciements. 
La larve est un cysticercoïde, de constitution plus simple que tous ceux décrits jusqu’à présent.

\section{Bibliographie}

Dickey (L. B.). - A new amphibian cestode. Journ. of parasitology, VII, 1921, p. $129-136$.

Fuhrmann (O.). - Die Tänien der Amplibien. Zool. Jahrb. Anat., IX, 1895, p. 207-226, pl. XVI.

Grassi (B.) et Rovelli (G.). - Ricerche enbriologiche sui Cestodi. Catania, in-4, 1892 , de $110 \mathrm{p}$. et IV pl.

Jewel (Minna E.). - Cylindrotænia americana nov. sp. from the cricket frog. Journ. of parasitology, II, 1916, p. 181-192.

Joyeux (Ch.). - Cycle évolutif de quelques cestodes. Recherches expérimentales. Bull. biol. France et Belgique, suppl. II, 1920, 219 p. et VII pl.

LëHE (M.). - Zur Kenntniss einiger Distomen. Zool. Anz. XXII, 1899, p. 524-539.

Laboraloire de Parasitologie de la Facullé de médecine de Paris. 